\title{
How can men be good allies for women in surgery? \#HeForShe
}

\author{
Douglas E. Wood
}

Department of Surgery, University of Washington, Seattle, WA, USA

Correspondence to: Douglas E. Wood. The Henry N. Harkins Professor and Chair, Department of Surgery, University of Washington, Box 356410, 1959 NE Pacific, BB-487, Seattle, WA 98195, USA. Email: dewood@uw.edu.

\begin{abstract}
Men have long been the dominant force in surgery, particularly in cardiothoracic surgery, and this has resulted in a tradition of a masculine culture that is not receptive to women. As a result, cardiothoracic surgery fails to recruit talented female surgeons, and with now over $50 \%$ of medical students being women, this means that cardiothoracic surgery potentially loses half of the physician talent pool. When women pursue a career in surgery, they face innumerable challenges and barriers, ranging from outright sexual harassment to daily microaggressions that demonstrate gender biases about perceived competence, work ethic, commitment, and professional ability. Women frequently suffer from unequal opportunities in clinical, academic, or leadership roles, and this can be further represented by disparities in compensation and time to academic promotion. Men have an outsized role in helping to provide a professional environment in surgery that is attractive and welcoming to women, and in supporting a culture that empowers equal opportunity for career success and advancement to both men and women. This important role of men is particularly true in cardiothoracic surgery since over $90 \%$ of the workforce are male. First and foremost, men can be critical allies to their female colleagues by actively supporting and promoting the women they work beside every day. Men are also important mentors for women, providing a safe space for frank conversations and career advice, with the sincere best interests of the female colleague at heart. Finally, men are important sponsors for women colleagues as well, talking about them, promoting them, and helping launch them into committee or leadership positions, while opening doors for clinical or academic advancement. The advantage of this work to men is the development of rewarding and lifelong relationships with female colleagues, and the benefit of their own reciprocal mentorship and allyship. Men have as much to gain as women from a modernization of our specialty and mitigation of gender disparities that undermine equal opportunity for career advancement. Men should not stand on the sidelines, and should be engaged and proactive as they work with women for gender equality in surgery. \#HeForShe
\end{abstract}

Keywords: Discrimination; harassment; professionalism; women in surgery; disparity; microaggression; gender

Submitted Jun 30, 2020. Accepted for publication Jul 28, 2020.

doi: $10.21037 /$ jtd-2020-wts-11

View this article at: http://dx.doi.org/10.21037/jtd-2020-wts-11

"A person may cause evil to others not only by bis actions but by bis inaction, and in either case be is justly accountable to them for the injury" -John Stuart Mill (1).

I was incredibly honored to be invited to participate in this special journal supplement on women in thoracic surgery. I have focused the most recent 10 years of my career on trying to address gender disparity in cardiothoracic surgery, and to look for ways that our specialty can be more receptive, inclusive, and progressive in providing an environment where both men and women can succeed and flourish in a thoracic surgical career. Therefore, it was easy for me to accept the invitation to write this piece in this special issue. It is a privilege to provide a male voice and perspective on the progress we have made in our specialty, and on the continued substantial disparities and opportunities for improvement in how women are treated, supported, and provided professional advances into leadership roles.

My initial enthusiasm was tempered as I considered the challenges and possible landmines of a middle-aged man 
writing an article about women's issues in their surgical careers. As I discussed with my wife and two daughters, I recognized the possibility, no actually the likelihood, of my getting something wrong, of having my own blind spots revealed in a published article, and the likelihood of being criticized by women who have had to navigate the career struggles in surgery that I have not personally faced.

My response, after breaking out into a cold sweat, was to dive deep into published research on gender disparities, sexual harassment, discrimination and abuse, as well as roadmaps for gender equality, with a plan of writing a highly scientific and referenced article. Hopefully, this would demonstrate rigorous analysis and perhaps protect my ego from my own exposed fallibilities on such a sensitive and nuanced subject. I read dozens of articles, took extensive notes, and planned an outline more rigorous than any scientific articles that I have written. But then I thought of all the incredible female allies who have helped teach me about the challenges they have had in their careers and inspire me with their strength, resilience, and leadership. I felt that it might be better to admit and expose my own vulnerabilities and simply write about what I have learned throughout the course of my career and from women who I count as valued colleagues. I know that I have blind spots. I am still learning. I have not lived the experience of my female colleagues in surgery, but I have learned many valuable lessons from them on how I can be a better mentor, ally, and sponsor for medical students, residents, junior colleagues, and peers.

One of the major things that men can do to support their women colleagues is to be a good ally. But it is equally critical to recognize how important women allies are for men in helping us recognize ingrained gender biases, identify microaggressions, and in so doing help us to be better colleagues and partners. A few years ago, I was at a small faculty dinner at a nice restaurant and part way through the meal I related an oft-told story regarding a visit that I made with my teenage daughter to New York City. This was a story I found very funny, partially because of the embarrassing circumstance of a father with his 14-year-old daughter enduring sexual-themed jokes in a Times Square comedy club. This is a story I had shared many times before and considered it edgy and self-deprecating, but never considered that others might find it offensive.

The next day one of my highly respected female colleagues took me aside and gently described how they were embarrassed and uncomfortable with my story and that they felt others may have been also. This was one of many important moments for me in learning about my own mistakes and in working to grow into a better male ally for my female colleagues. I paused, and thought deeply about the feedback that I was receiving from my colleague and friend. She provoked an epiphany about how my words and actions are perceived by others. I apologized to my female colleague and thanked her for having the courage to speak up and to educate me, and also thanked her for having the respect for me to take the initiative to give that feedback. I have never told that story again. And in my head, I did a rewind of multiple stories and events throughout my career that I was suddenly ashamed of. I have told jokes, laughed at stories, and have made insensitive comments that when I look backwards, I find cringe-worthy. Because of a trusted female colleague's willingness to help me, I am more aware of the quietest and potentially most marginalized person in the room. While I know I still make mistakes, her feedback has helped me be more sensitive and a better colleague for the women I work with. This is only one of innumerable anecdotes of strong and influential women who have helped me be a better leader and ally. Gender disparity in cardiothoracic surgery is self-perpetuating, creating an environment that does not appear receptive to women due to sexual harassment, discrimination, microaggressions, and limited female role models. Men have an outsized role to recognize, speak out, and lead change in surgical careers that afford women equal opportunity to succeed and have rewarding professional careers.

\section{Gender disparity in cardiothoracic surgery}

Cardiothoracic surgery is a tremendous specialty but also has one of the highest gender disparities of any medical field. We have been a macho specialty with a swagger of testosterone and overconfidence that, until recently, was very unreceptive to women (2). In the United States, just $6 \%$ of cardiothoracic surgeons were women until a few years ago. While more recently this ratio has grown to approximately $10 \%$, there is still woeful gender disparity that should be a call to action for all of us within cardiothoracic surgery. Our specialty will be stronger, our care of patients will be better, and we will better attract the strongest and most capable students and residents if we evolve to equally support careers of women and men in cardiothoracic surgery. Further, many of the most effective leadership traits in our current environment of healthcare are demonstrated particularly by women leaders. Not only should our efforts be to recruit women into cardiothoracic surgery as a 
specialty, but we should also be deliberate in our support of women advancing to levels of leadership within our hospitals, departments, and national specialty societies (2). However, women face disproportionate challenges of sexual harassment, discrimination, and microaggressions that exact a heavy toll, a "gender tax" that stifles their inclusion and advancement in their surgical careers.

\section{Sexual harassment}

Sexual harassment includes unwanted sexual advances, requests for sexual favors and other unwelcome conduct that is sexual in nature. It also includes situations in which the work or study environment is made intimidating or offensive as a result of actions that are gender-based. The causes and prevalence of sexual harassment in medicine was well-described in a recent publication from the National Academies of Sciences, Engineering, and Medicine (3).

"Academic science, engineering, and medicine exhibit at least four characteristics that create higher levels of risk for sexual harassment to occur: a. Male-dominated environment, with men in positions of power and authority. b. Organizational tolerance for sexually harassing behavior (e.g., failing to take complaints seriously, failing to sanction perpetrators, or failing to protect complainants from retaliation). c. Hierarchical and dependent relationships between faculty and their trainees (e.g., students, postdoctoral fellows, residents). d. Isolating environments (e.g., labs, field sites, and hospitals) in which faculty and trainees spend considerable time. 2. Sexual harassment is common in academic science, engineering, and medicine. Each type of sexual barassment occurs within academic science, engineering, and medicine at similar rates to other workplaces. a. Greater than 50 percent of women faculty and staff and 20-50 percent of women students encounter or experience sexually barassing conduct in academia. $b$. Women students in academic medicine experience more frequent gender harassment perpetrated by faculty/staff than women students in science and engineering. c. Women students/trainees encounter or experience sexual barassment perpetrated by faculty/staff and also by other students/ trainees. $d$. Women faculty encounter or experience sexual barassment perpetrated by other faculty/staff and also by students/ trainees. e. Women students, trainees, and faculty in academic medical centers experience sexual harassment by patients and patients' families in addition to the harassment they experience from colleagues and those in leadership positions."

Although men can also be subject to sexual harassment, this form of workplace intimidation and bias is predominantly experienced by women, creating a hostile work environment and substantial obstacles to advancement, which are often exacerbated by power differentials (4). Even today, sexual harassment is commonly inflicted on female surgeons and surgical trainees. A 2018 survey administered through the American College of Surgeons and the Association of Women Surgeons revealed that $58 \%$ of women had experienced sexual harassment in the previous twelve months, with female trainees more than twice as likely to experience harassment compared to female attending surgeons (5). Extreme forms of sexual harassment also include actual assault. We would like to think that this doesn't occur, but unfortunately the workplace environment and the historical culture within medicine and surgery provide both opportunity and tacit tolerance of this behavior. Call rooms and overnight shifts create the opportunity and setting for sexual assault. Intense shared clinical experiences, personal isolation, and a historically male-dominated culture fuels the potential for assault or harassment. Finally, an ethos of toughness and self-reliance in surgery, combined with fears of retaliation, create barriers to reporting and disciplinary remediation when incidents of sexual harassment occur.

While physical sexual assault is the most obvious and aggressive form of harassment, unwanted sexual attention is the most common form, including unwelcome verbal or physical sexual advances. The substantial power hierarchy in medicine also creates opportunity for sexual coercion when favorable professional or educational treatment is conditioned on sexual activity. However, harassment can also be more subtle with verbal or nonverbal behaviors that convey hostility, objectification, exclusion, or secondclass status relating to gender. It is likely that we have all witnessed or perhaps even participated in this more insidious type of behavior. It might have been something as seemingly innocuous as a comment on appearance or clothing, or a gender-biased comment relating to some aspect of professional competence.

Sexually harassing behavior can be either direct (targeted at an individual) or ambient (a general level of sexual harassment in an environment) and is harmful in both cases. It creates a hostile environment when gender harassment or unwanted sexual attention is severe or pervasive enough to interfere with one's work performance and mental health, or impede one's ability to get an education or when it is quid pro quo sexual harassment (6).

The persistent specter of sexual harassment is an additional workplace burden that our female colleagues carry daily, an extra weight of navigating an already 
complex and intense professional environment that requires women to think carefully about their dress, their words, and potential secondary agendas in their professional interactions with male colleagues (7). This is a burden that most men do not need to face, and therefore is easily overlooked and underappreciated by even well-meaning male colleagues.

So what can we do? A quote from a woman in medicine put it succinctly, "do not rape us, and don't think that we want to sleep with you." The first part seems pretty straightforward; it is likely we can have universal agreement that physical sexual assault should be a never event and that it is completely intolerable behavior. The potential for sexual attraction and interest is more complex and more nuanced, but can also probably be addressed with some fairly straightforward principles. For professional peers who are single it may be reasonable to consider dating if there is mutual interest, albeit with cautions about the potential changes in professional relationships. A key principle is embedded in the term "mutual interest", meaning that there is clear bilateral consent in flirting, dating, and pursuing a possible relationship. If one of the parties demonstrates their lack of interest, it is critically important that the other party step back and avoid further romantic approaches and work to reestablish and maintain an effective professional relationship. Continued pursuit of a relationship, even amongst peers, creates a difficult and hostile work environment that disproportionately harms women.

Male peers can be important supporters and allies for their female colleagues when they observe unwanted sexual advances from others, incidences of gender bias, or other hostile behaviors. It is critical that men be alert and observant for harassment that they witness directed towards their female colleagues. Depending on the situation, it may be helpful to check-in with the colleague after the incident, or to speak up in the moment to externally demonstrate recognition of and intolerance for gender harassment. In more serious cases male allies should be proactive in helping to report hostile behavior to a chief, chair, or human resource personnel (8).

Senior surgeons and those in positions of authority have the same responsibilities to support women as described above, but with an added duty of supporting and protecting the women in their department, of role modeling a culture of inclusion and intolerance of gender harassment, and of demonstrating accountability to definitively address and discipline acts of harassment or unprofessional behavior when they occur. First and foremost, senior surgeons need to start with the principal noted above, "don't think that we want to sleep with you." Stated simply, surgeons in positions of authority cannot, under any circumstance, demonstrate or act on sexual attraction or interest in a more junior female colleague. While the emotion may be sincere, with no intent of coercion, the hierarchy and power imbalance create the potential, interpretation, or appearance of obligation, placing the junior female colleague in an untenable position and the senior male surgeon at risk of disciplinary action.

Some surgeons have over-interpreted this principle, taking on a highly defensive posture in their relation to female colleagues. In an effort to appear above reproach and with the intent of preventing any opportunity for allegations of sexual harassment, some surgeons have chosen to avoid one-on-one meetings or social interactions with women students or colleagues. While well-intended, this approach generates a whole different form of gender discrimination. First, avoidance of private meetings and confidential conversations with female students or colleagues denies them important networking and relationship-building opportunities, as well as the benefit of senior surgeon advice and mentorship (9). Second, this philosophy infers a dangerous, harmful, and incorrect view that women are prone to infer sexual intent or to threaten men with allegations of inappropriate advances. Finally, such a selfdefensive principle with women undermines important trust that is critical for establishing strong professional relationships that foster opportunities for support, mentorship, and sponsorship.

As male surgeons, we may receive reports of sexual harassment, both observed and experienced, from our female colleagues. Sometimes this instance will be solely to share a problem with a friend and gain moral support, and sometimes it may be seeking advice on what to do or perhaps assistance or official reporting. As friends and colleagues, what is most important is to listen, to demonstrate sincere empathy, and not necessarily assume that we are going to "fix" the problem. However, we also should not shy away from the difficult challenges faced by our women colleagues. We should show courage and a willingness to encourage and support women to report serious or ongoing incidents of harassment, as well as a commitment to stand in solidarity with them when they are threatened.

Senior surgeons in positions of authority are tasked with the important responsibility of responding to incidences of sexual harassment or other forms of gender bias that 
create a hostile work environment. These cases can be extremely challenging, sometimes pitting a politically and economically powerful senior male surgeon against a relatively junior female student or early career surgeon. This is a scenario in which institutions have historically failed by protecting the powerful individual and the status quo, allowing a lack of accountability for sexual harassment or other unprofessional behavior. However, strong leaders will show courage in the face of holding a powerful male colleague accountable for unprofessional behavior. It is critical to recognize the importance of protecting and promoting female colleagues by providing a professional environment where they can work safely and without the specter of bullying, harassment, or retaliation.

When senior leaders are faced with relatively minor complaints, these can possibly be handled with direct feedback to the offending party while assuring protections of confidentiality and prohibition against retaliation. For more serious offenses, most of senior leadership are not equipped with the professional tools of adequate investigation, or the appearance of impartiality. In these situations, it is important to rely upon an official institutional investigation that can provide findings and recommendations to help surgeon leaders determine disciplinary action, policy changes, and communication. One factor that is a common challenge is the impact that confidentiality has on feedback and communication to the victim, department, or institution. This can result in the incorrect perception that little has been done to respond to serious complaints of harassment. To rectify this, we should work to establish a system that both respects the confidentiality of the individuals involved and communicates our policies and actions against sexual harassment.

\section{Gender microaggressions}

The professional environment in surgery is also fraught with a wide variety of gender-based microaggressions. These actions include both verbal and nonverbal behaviors that are sometimes obvious, but more frequently are subtle and insidious in communicating negative, hostile, or derogatory messages that marginalize our female colleagues. These actions can be both intentional and unintentional, and most commonly are not acknowledged, yet occur frequently in day-to-day interactions. Some that may be more obvious and related to the previous section on sexual harassment involve sexual objectification, sexist humor and jokes, and use of sexist language. More subtle, but no less damaging, are implications of gender inferiority represented by "mansplaining", men co-opting ideas from female colleagues, men speaking over women during group meetings, and undermining assertive women by labeling them with sexist stereotypes ("bitchy", "bossy", or "aggressive"). These microaggressions also include the devaluing of women's professionalism through in the frequent use of casual first names in introductions of female surgeons while male surgeons are more often referred to with formal professional attributions including the title "Doctor". This differential treatment often occurs within the same sentence (10). Male colleagues who wish to be good allies for the women that they work with should be aware of these common microaggressions in order to avoid unintentionally harming their female colleagues, as well as being alert to redirect or speak up when they are witnessed.

Observe the next faculty or committee meeting you are attending and pay attention to who is speaking. In many professional settings it has been noted that men tend to overestimate their abilities and display overconfidence whereas women tend to do the opposite, and this finding is usually also represented by speaking times in group meetings. Women are usually a significant minority in these meetings, particularly in our specialty of cardiothoracic surgery. With a little quiet observation, one can note that men generally do most of the talking and may even step in and interrupt or speak over when a woman is talking. These are generalities that are not uniformly true, but I encourage men to pay attention in the meetings that they attend in order to be aware, avoid the issues themselves, and to serve as an ally to the woman or women in the room. For example, male allies could call on a woman to express her opinion or redirect a discussion back to a woman who has just been interrupted by a male colleague.

Another common form of microaggression is "mansplaining", the pejorative term describing situations where a man explains something to a woman in a condescending, overconfident, or oversimplified manner. It is painful to say that almost certainly all of us men have mansplained to our female colleagues at some time or another, and may continue to do so. This can occur in the professional domain of describing clinical conditions, technical aspects of operations, or other patient issues to equally-qualified female colleagues. However, it can also commonly extend to more casual domains in discussions of politics, economy, social issues, or sports. Most of the time this is unintentional, yet the unspoken premise is gender bias that infers professional or intellectual inferiority and 
the need for a man's explanation to improve a women's "understanding". More aggressive forms of mansplaining are intentional with comments made in a hostile or condescending manner, and may miss the irony that often the explainee knows more than the explainer. Advice for men hoping to avoid this pitfall could be to pause and think before launching into an explanation to a female colleague, possibly ask questions to solicit the female colleague's own views, or sometimes simply to keep quiet and not assume an explanation is needed when you are not asked. As allies we can also be observant when our female colleagues are subject to condescending explanations or an undervaluing of their own knowledge and ideas. A good ally can provide an antidote to mansplaining by turning questions back to our female colleagues and helping them reassert their professionalism and professional competence.

Gender social norms and concerns about allegations of sexual harassment easily create an unintended form of microaggression consisting of male colleagues avoiding or being overly cautious about meetings with women. This caution may extend to an avoidance of the casual social networking that is a common foundation in solidifying professional relationships. Simply stated, men ask male colleagues to share a beer, watch a sporting event, or otherwise share outside social time. This is normal and forges a social bond that strengthens the professional relationship as well, and affords a high level of trust and intimacy that is mutually supportive and professionally advantageous. Women may be intentionally or unintentionally excluded from these unofficial networks and lose the career and social advantages gained from the networking outside of the office. This situation further disadvantages women who already may have trouble obtaining one-on-one appointments with senior colleagues due to a hypervigilance of avoiding potential charges of sexual harassment, as noted earlier.

It is unclear why women surgeons are so commonly referred to by their first names in professional meetings and introductions while their male peers usually maintain a more formal introduction with their professional title (10). This is another example of implied gender-based inferiority that is subtle yet insidious in undermining women surgeons' status in their career and in their institution. Once recognized, this problem is a simple one to address. I encourage male colleagues to pay attention to this issue and purposely assure that they are introducing and addressing their female colleagues with their professional title when appropriate. If anything, it is worth over correcting given how pervasive this habit has become in our professional environments. Of course, this principle needs to be balanced with pragmatism of using casual introductions in casual environments but assuring professional introductions for all colleagues in front of patients, when introducing a speaker, and in our more formal settings.

These microaggressions are examples of daily challenges our female colleagues in cardiothoracic surgery are required to navigate and that males in our specialty at best may not recognize, or at worst may be intentional offenders. Males make up $90 \%$ of the workforce in cardiothoracic surgery and therefore have the dominant role in trying to individually avoid actions that undermine female colleagues (11). Men can work to be observant for harassment and discrimination directed at women surgeons who we work with, and in being willing to speak up as a supportive colleague and ally. As noted above, some of our actions can simply be subtle redirection of conversations within meetings and being deliberate in recognizing our female colleagues and calling on them for their opinions. More serious or intentional slights require the courage of speaking up, even against powerful male colleagues, to call out bad behavior or gender bias. Finally, sometimes a good ally is also simply a good friend, where a quiet word of recognition, understanding, and support can demonstrate empathy and solidarity with our female colleagues.

\section{What can men do?}

Men can have different roles in supporting women in cardiothoracic surgery depending on their own seniority and position within the surgical hierarchy. Male medical students and residents have few opportunities to actively promote their female peers, but have important roles in being good colleagues and being supportive allies that can help avert or mitigate gender harassment or bias that they witness (Table 1). Male students and residents may be in the best position to observe problematic interactions tolerated by their female colleagues and provide individual personal support for minor infractions, or formal reporting and for more serious incidents. Leadership roles, research opportunities, and clinical training experiences are influenced by the informal network amongst students or residents where men can be attentive to ensuring equal opportunity for everyone in their group and the avoidance of overt or covert gender discrimination.

Make sure that women peers have a chance to speak during conferences and meetings; redirect back to them if 
Table 1 Dos and Don'ts for male surgery residents and early career faculty

\begin{tabular}{ll}
\hline Dos & Don'ts \\
\hline - Include women colleagues in out of work social functions & - Develop or support a "bro culture" at work \\
- Invite women to express their opinion in meetings & - Tell stories or jokes relating to sex \\
- Suggest women to be in included in committees, panels, etc. & - Talk over women in conferences or meetings \\
- Use formal title of "Doctor" when introducing women & - Introduce women colleagues by their first name in formal \\
colleagues & introductions \\
- Be alert for harassment or bias directed at women & - Accept gender biases regarding professional competence \\
- Offer support to women subject to bias or harassment & - Disparage or resent gender accommodations around pregnancy \\
- Speak up about discrimination or harassment & Belittle family-friendly policies and schedules that improve \\
& Be a trusted confidant and friend \\
Be a leader and ally for policies and schedules that improve
\end{tabular}

they are interrupted or overshadowed by male colleagues. Include women equally in the informal social networking amongst peers. Try to assure that they are not excluded from the typical "male bonding experiences" of drinks together after work, watching sports, or weekend functions like a group hike or dinner. Finally, in a male-dominated environment like surgery, it is easy to develop a "bro culture" and crude behavior or jokes, commonly referred to a "locker room humor" (12). This culture certainly creates an unpleasant or even hostile environment for women students and surgeons that may be underappreciated because of their minority status and fear of speaking out. Research would suggest that a male-dominated culture is mitigated once there is approximately one third of women in the workplace. Since cardiothoracic surgery is far from that critical mass of women, it is incumbent upon men to try to speak up and actively overcome this environmental aspect of gender harassment (8).

Early and midcareer surgeons can learn an incredible amount from their female colleagues while having a profoundly supportive and positive influence on the women surgeons they work with. In addition to the wealth of knowledge and experience that our female colleagues bring to clinical care and decision-making, they add diversity and often provide important alternative viewpoints on critical aspects of departmental policies, ethical issues, interdepartmental turf battles, and surgical education. Male surgeons benefit from female allies who they can turn to for advice and support, and who often provide a new solution to a problem or an observation that helps us better support our female peers and patients.

Look for opportunities for your female colleagues to be showcased in a meeting, on a panel, on a committee, or in a leadership position within the hospital or surgical society. Avoid organizing or participating in a "manel", an all-male expert panel, when there are many well-qualified women and will add to the diversity and strength of the panel (13). Be alert for gender discrimination or subtle acts of bias. Be willing to speak up and speak out to support the women that you work with. Show up for meetings about gender issues. Show that you are interested. Show that you can listen. Most importantly, do not feel threatened or feel that women are advantaged in promotion or leadership decisions (11).

Senior surgeons may have political power and influence related to their stature in the department and hospital, their economic power, and their development of relationships in the healthcare system. Some senior surgeons may also wield the power of specific leadership positions as division chiefs, department chairs, medical school deans, or other hospital leadership positions. These individuals have an enormous influence on the culture of gender equity and diversity in their environment, as well as on individual female students, residents, and faculty in their department (Table 2).

At the organizational level, encourage the development of a women's group or Women's Council, both to support the networking of women surgeons with each other, but also to provide a forum for evaluating the environment, identifying problems and barriers for women surgeons, 
Table 2 Dos and Don'ts for male senior surgeons and leaders

\begin{tabular}{|c|c|}
\hline Dos & Don'ts \\
\hline $\begin{array}{l}\text { Role-model professional behavior that avoids sexist remarks and } \\
\text { culture }\end{array}$ & Avoid one-on-one meeting with women \\
\hline Provide mentorship and coaching to female colleagues & Accept male only committees, panels, leadership \\
\hline $\begin{array}{l}\text { Develop Women's and/or Diversity Council to identify barriers and } \\
\text { advise on policies and actions to improve diversity and inclusion }\end{array}$ & Allow compensation or other disparities to go uncorrected \\
\hline Evaluate compensation and promotion data for gender disparity & Cover up or avoid dealing with sexual harassment \\
\hline $\begin{array}{l}\text { Find trusted female allies to lean on for advice } \\
\text { Be engaged in gender issues, join groups, attend symposia, read, } \\
\text { educate yourself }\end{array}$ & Flirt or demonstrate sexual interest \\
\hline \multicolumn{2}{|l|}{$\begin{array}{l}\text { Develop schedules and technology to support work-life integration (e.g., } \\
\text { Zoom access, avoidance of early and late meetings) }\end{array}$} \\
\hline \multicolumn{2}{|l|}{ Assure good parental leave policies } \\
\hline Assure lactation access and accommodation & \\
\hline
\end{tabular}

providing important advice to senior leadership, and recommending policies to improve the workplace for both men and women. Senior surgical leaders are likely to be made more aware of and engaged with gender issues in surgery by getting involved in women's organizations and groups. Examples of groups to become involved in include: Women in Thoracic Surgery and the Association of Women Surgeons. In our home institutions there are likely seminars or programs related to women in science or women in medicine that are also valuable for male surgeons, especially in senior roles. For male surgeons involved in social media, following some of these women's groups or prominent female surgical leaders affords yet another way to learn about gender related issues, barriers, and problems in surgery.

Senior surgical leaders are most commonly in the position of recruitment and promotion. This is an opportunity to deliberately identify and seek out highly qualified women for faculty positions and leadership roles, which then also helps to recruit and retain female medical students and residents interested in surgery. However, an important factor in successfully recruiting and promoting women is assuring that the department confirms equity and fairness, and that the environment is welcoming and supportive of women surgeons. First, it is critical to assure gender parity in compensation, academic promotion, and leadership development and opportunity. This point requires a willingness to look specifically at data around compensation and promotion, identifying disparities where they exist, and actively fixing them. Second, work schedules, departmental activities, and environmental culture should recognize and be supportive of work-life balance that supports all individuals in their personal and family activities. While this is equally true for men and women, it is more often that women pay attention to these issues within the workplace and are potentially discriminated against because of an outdated viewpoint of "work ethic" that overshadows the individual and/or their family. Both men and women benefit from family-friendly policies and actions that include consideration of appropriate meeting times, options for remote meeting attendance, family leave policy, and flexibility in work and call schedules. This includes gender specific accommodations around pregnancy and lactation along with the gender neutral accommodations around parental leave.

A difficult area for senior leaders to navigate relates to the delicate balance between female inclusion in various committees, groups, or projects versus an appearance of 
tokenism or pressure for women to participate in roles they may not want. Well-intended efforts to develop a Women's Council or Diversity Council may disproportionately direct women into these roles, particularly in cardiothoracic surgery where there may be very few women to join and share in these responsibilities. It is important to realize that our female colleagues have their own portfolio of interests and expertise that they wish to prioritize in their career, and that these may not be enhanced by filling gender role needs of the department or institution. Further, it is common that many of these roles are "volunteer" and do not come with specific financial support in the form of allocated time or additional compensation. Therefore, if not a sincere interest of the faculty member, these responsibilities actually becomes another form of "gender tax" that places an additional and unwanted burden on a woman's surgeon who wishes to direct her priorities elsewhere.

Finally, senior surgical leaders also have the most influence over the physical environment, which indirectly represents the values, culture, and inclusiveness of the surgical department. Hallways and walls of the surgical department reinforce surgery as a male-dominated specialty. This is not necessarily easy to change when the hallways are covered with paintings or photographs of previous faculty and graduating residents who, until recently, were almost exclusively male. However, this is another area for senior leaders can listen to and learn from more junior colleagues and examine creative ways of modifying the external environment to better represent a desired culture of improved diversity and inclusion.

\section{Summary}

Men have long been the dominant force in surgery, particularly in cardiothoracic surgery, and this has resulted in a tradition of a masculine culture that is not receptive to women. As a result, cardiothoracic surgery fails to recruit talented female surgeons, and with now over $50 \%$ of medical students being women, this means that cardiothoracic surgery potentially loses half of the physician talent pool. When women pursue a career in surgery, they face innumerable challenges and barriers, ranging from outright sexual harassment or assault to daily microaggressions that demonstrate gender biases about perceived competence, work ethic, commitment, and professional ability. Women frequently suffer from unequal opportunities in clinical, academic, or leadership roles, and this can be further represented by disparities in compensation and time to academic promotion.

Men have an outsized role in helping to provide a professional environment in surgery that is attractive and welcoming to women, and in supporting a culture that empowers equal opportunity for career success and advancement to both men and women. This important role of men is particularly true in cardiothoracic surgery since over $90 \%$ of the workforce are male.

First and foremost, men can be critical allies to their female colleagues by actively supporting and promoting the women they work beside every day. Men are also important mentors for women, providing a safe space for frank conversations and career advice, with the sincere best interest of the female colleague at heart. Finally, men are important sponsors for women colleagues as well, talking about them, promoting them, and helping launch them into committee or leadership positions, while opening doors for clinical or academic advancement. The advantage of this work to men is the development of rewarding and lifelong relationships with female colleagues, and the benefit of their own reciprocal mentorship and allyship. The benefit to the specialty of cardiothoracic surgery is an increase in recruitment of talented surgeons, an improvement in our professional culture, enhancement and innovation in our field, and a diversity that better represents the patients that we care for. Men have as much to gain as women from a modernization of our specialty and mitigation of gender disparities that undermine equal opportunity for career advancement. Men should not stand on the sidelines, and should be engaged and proactive as they work with women for gender equality in surgery. \#HeForShe

\section{Acknowledgments}

Funding: None.

\section{Footnote}

Provenance and Peer Review: This article was commissioned by the Guest Editors (Cecilia Pompili and Leah Backhus) for the series "Women in Thoracic Surgery" published in Fournal of Thoracic Disease. The article was sent for external peer review organized by the Guest Editors and the editorial office.

Conflicts of Interest: The author has completed the ICMJE uniform disclosure form (available at: http://dx.doi. org/10.21037/jtd-2020-wts-11). The series "Women in 
Thoracic Surgery" was commissioned by the editorial office without any funding or sponsorship. The author has no other conflicts of interest to declare.

Ethical Statement: The author is accountable for all aspects of the work in ensuring that questions related to the accuracy or integrity of any part of the work are appropriately investigated and resolved.

Open Access Statement: This is an Open Access article distributed in accordance with the Creative Commons Attribution-NonCommercial-NoDerivs 4.0 International License (CC BY-NC-ND 4.0), which permits the noncommercial replication and distribution of the article with the strict proviso that no changes or edits are made and the original work is properly cited (including links to both the formal publication through the relevant DOI and the license). See: https://creativecommons.org/licenses/by-nc-nd/4.0/.

\section{References}

1. Daar DA, Abdou SA, Wilson SC, et al. A call to action for male surgeons in the wake of the \#MeToo movement. Ann Surg 2019;270:26-8.

2. Wood DE. Take it to the limit. Ann Thorac Surg 2014;98:1893-901.

3. National Academies of Sciences, Engineering, and Medicine 2018. Sexual Harassment of Women: Climate, Culture, and Consequences in Academic Sciences, Engineering, and Medicine. Washington, DC: The National Academies Press. Available online: https://doi. org/10.17226/24994

4. Miller P. \#MeToo in Surgery: Narratives by Women Surgeons. Narrat Inq Bioeth 2019;9:179-83.

Cite this article as: Wood DE. How can men be good allies for women in surgery? \#HeForShe. J Thorac Dis 2021;13(1):492501. doi: 10.21037/jtd-2020-wts-11
5. Nayyar A, Scarlet S, Strassle PD, et al. A national survey of sexual harassment among surgeons. Available online: https://www.asc-abstracts.org/abs2019/85-06-a-nationalsurvey-of-sexual-harassment-among-surgeons/. Accessed June 27, 2020.

6. National Academies of Sciences, Engineering, and Medicine 2018. Sexual Harassment of Women: Climate, Culture, and Consequences in Academic Sciences, Engineering, and Medicine. Washington, DC: The National Academies Press. Available online: https://doi. org/10.17226/24994

7. Jagsi R. Sexual Harassment in Medicine - \#MeToo. N Engl J Med 2018;378:209-11.

8. Freischlag JA, Faria P. It Is Time for Women (and Men) to Be Brave: A Consequence of the \#MeToo Movement. JAMA 2018;319:1761-2.

9. Byerly JS. Mentoring in the era of \#MeToo. JAMA 2018;319:1199-1200.

10. Files JA, Mayer AP, Ko MG, et al. Speaker Introductions at Internal Medicine Grand Rounds: Forms of Address Reveal Gender Bias. J Womens Health (Larchmt) 2017;26:413-9.

11. Jain S, Madani K, Flint L, et al. What Does It Mean to Be a Male Ally? Implementing Meaningful Change in Gender Representation in Medicine. J Am Coll Surg 2020;230:355-6.

12. Petty J, Muzzey ME, Maas FK, et al. \#HowWillIChange: Engaging men and boys in the \#MeToo movement. Psychol Men Masc 2019;20:612-22.

13. Wilcox AR, Trooboff SW, Lai CS, et al. Trends in Gender Representation at the American College of Surgeons Clinical Congress and the Academic Surgical Congress: A Mixed Picture of Progress. J Am Coll Surg 2019;229:397-403. 